\title{
Neural mechanisms of the rejection-aggression link
}

\section{David S. Chester, ${ }^{1}$ Donald R. Lynam, ${ }^{2}$ Richard Milich, ${ }^{3}$ and C. Nathan DeWall ${ }^{3}$}

\author{
${ }^{1}$ Department of Psychology, Virginia Commonwealth University, Richmond, VA 23284, USA, ${ }^{2}$ Department of \\ Psychological Sciences, Purdue University, West Lafayette, IN 47907, USA, and ${ }^{3}$ Department of Psychology, \\ University of Kentucky, Lexington, KY 40506, USA
}

Correspondence should be addressed to David S. Chester, Department of Psychology, Virginia Commonwealth University, 808 West Franklin St., Richmond, VA 23284, USA. E-mail: dschester@vcu.edu

\begin{abstract}
Social rejection is a painful event that often increases aggression. However, the neural mechanisms of this rejectionaggression link remain unclear. A potential clue may be that rejected people often recruit the ventrolateral prefrontal cortex's (VLPFC) self-regulatory processes to manage the pain of rejection. Using functional MRI, we replicated previous links between rejection and activity in the brain's mentalizing network, social pain network and VLPFC. VLPFC recruitment during rejection was associated with greater activity in the brain's reward network (i.e. the ventral striatum) when individuals were given an opportunity to retaliate. This retaliation-related striatal response was associated with greater levels of retaliatory aggression. Dispositionally aggressive individuals exhibited less functional connectivity between the ventral striatum and the right VLPFC during aggression. This connectivity exerted a suppressing effect on dispositionally aggressive individuals' greater aggressive responses to rejection. These results help explain how the pain of rejection and reward of revenge motivate rejected people to behave aggressively.
\end{abstract}

Key words: aggression; social rejection; reward; frontostriatal; fMRI

\section{Introduction}

It is not hard to imagine how an individual, pushed against their will to the fringes of a community, might react violently towards the perceived sources of such pain. Yet our understanding of the precise neural mechanisms that link experiences of rejection to aggressive retaliation is imperfect. In what follows, we summarize a brain imaging project that sought to understand the biological and psychological forces that drive the rejection-aggression link. Specifically, we tested whether the neural regulation of the pain of rejection magnified the subsequent 'sweetness' or reward of taking revenge upon the source of rejection. We then tested whether this interplay between regulatory and reward reactivity was linked to greater severity of the aggressive retaliatory response. Building on this, we tested whether this mechanism helped explain why some people tend to be more dispositionally aggressive than others.

\section{The rejection-aggression link}

Social rejection occurs when active attempts at social inclusion and belonging are rebuffed by the targets of affiliative acts (Williams, 2009). Such experiences are pervasive and have longterm adverse effects on human health. Indeed, social rejection is reliably linked to greater anxiety, depression and learned helplessness (Leary, 1990; Williams, 2009). These internalized consequences of rejection are juxtaposed against this phenomenon's more externalizing consequences, such as aggression. Social rejection is one of the most well-established causes of aggression (Leary et al., 2006; Ren et al., 2018). In laboratory settings, rejected individuals exhibit heightened aggressive behavior towards both their rejecters and innocent bystanders (Twenge et al., 2001; Buckley et al., 2004). As evidence that this phenomenon extends out into the broader world, experiences of rejection are thematic among the lives of the majority of

Received: 3 October 2017; Revised: 25 February 2018; Accepted: 19 March 2018

(c) The Author(s) (2018). Published by Oxford University Press.

This is an Open Access article distributed under the terms of the Creative Commons Attribution Non-Commercial License (http://creativecommons.org/ licenses/by-nc/4.0/), which permits non-commercial re-use, distribution, and reproduction in any medium, provided the original work is properly cited. For commercial re-use, please contact journals.permissions@oup.com 
school shooters (Leary et al., 2003). Investigations into the psychological processes that motivate the rejection-aggression link have revealed anger (Chow et al., 2008), hostile cognitive biases (DeWall et al., 2009), disinhibition (Rajchert and Winiewski, 2016), non-adherence to societal norms (Poon and Teng, 2017) and desires to re-establish feelings of control (Warburton et al., 2006; Wesselmann et al., 2010), as likely motivations. Although the psychological mechanisms of this effect are becoming well explicated, the neural mechanisms of the rejection-aggression link remain largely unexamined.

\section{Neural correlates of rejection}

Rejection threatens the fundamental human need for social connections (MacDonald and Leary, 2005). Social Pain Overlap Theory posits that the brain evolved to respond to this social injury with a broad and powerful recruitment of multiple neural systems that include brain regions critical to the experience of pain (Eisenberger and Lieberman, 2004).

Social pain: DACC and anterior insula. As outlined in Social Pain Overlap Theory, social pain is the aversive, affective and somatic response to perceptions of social rejection (Eisenberger and Lieberman, 2004; Eisenberger, 2012). As evidence of this, functional neuroimaging has associated experimental inductions of exclusion with activity in brain regions associated with the affective component of pain: anterior insula, dorsal anterior cingulate cortex (DACC; Eisenberger et al., 2003; Eisenberger, 2012; Rotge et al., 2014). Reactivity of the DACC to rejection is associated with greater retaliatory aggression, though only among individuals with relatively poor executive functioning (Chester et al., 2014). This finding offers initial evidence that the brain's social pain response to rejection may exert a motivating influence on subsequent aggressive retaliation.

Regulation of social pain: VLPFC. Social pain, like physical pain, is not an unchecked response to stimuli. Instead, robust regulatory mechanisms exist to modulate neural pain reactivity (Price, 2000). Numerous studies point to the ventrolateral prefrontal cortex's (VLPFC) role in responding to socially painful events (Eisenberger et al., 2003; Chester and DeWall, 2014). In this context, the VLPFC exerts a regulatory role that inhibits the subjective experience of pain by inhibiting brain regions that generate the distressing experience of pain (Eisenberger et al., 2003; Yanagisawa et al., 2011; Kawamoto et al., 2012). These findings fit with a much larger literature demonstrating the critical role of the VLPFC in regulating distress and negative affect (Wager et al., 2008).

Affect regulation is one of an array of functions in the VLPFC, which largely involve inhibition (Aron et al., 2014). Indeed, the VLPFC is a relatively large and heterogeneous region of the prefrontal cortex that is anatomically and functionally heterogeneous (Levy and Wagner, 2011). Most posterior regions that are close to the motor regions of the frontal lobe tend to facilitate the inhibition of concrete motor and behavioral responses (pars opercularis/triangularis; Levy and Wagner, 2011; Aron et al., 2014), whereas more anterior VLPFC regions tend to subserve the inhibition and regulation of more abstract emotional and cognitive processes (pars triangularis/orbitalis; Wager et al., 2008; Buhle et al., 2014). Yet how might VLPFC recruitment during rejection impact the neural underpinnings of retaliatory aggression?

\section{Neural correlates of retaliatory aggression}

Retaliatory aggressive behavior is associated with greater activity in a host of cortical regions during the act (dorsomedial PFC, posterior cingulate cortex, anterior and posterior insula; Krämer et al., 2007; Lotze et al., 2007; Dambacher et al., 2014; Chester and DeWall, 2016; Emmerling et al., 2016). Germane to this project, retaliatory aggression has also been linked to activity in the ventral striatum during the aggressive act, a neural region reliably linked to the experience of pleasure and reward (Chester and DeWall, 2016). The VLPFC exerts a robust regulatory influence on the ventral striatum, the connectivity of which predicts greater self-regulatory success (e.g. Wagner et al., 2013). Retaliatory aggression is associated with reduced connectivity between the ventral striatum and the VLPFC, potentially indicating a dysregulated reward response (Buades-Rotger et al., 2016; Chester and DeWall, 2016; Chester, 2017). These findings suggest that a likely neural mechanism underlying retaliatory aggression is a magnified and dysregulated striatal response, that may serve to reinforce such aggressive acts. Our results further fit with psychological research that implicates reward and pleasure as central components of revenge-seeking tendencies (Chester and DeWall, 2018b). The pleasure of retaliation likely motivates aggression in both prospective and concurrent manners, with individuals seeking out acts of retaliatory aggression for the anticipated and currently felt rewards it brings. However, it remains unknown how such striatal mechanisms interact with neural responses to rejection.

\section{The excessive recruitment model}

Conventionally, VLPFC recruitment during aversive experiences is theorized to be an adaptive regulatory response (Wager et al., 2008). Indeed, self-regulation failures (e.g. aggression) that occur because of aversive experiences (e.g. rejection) are thought to arise from an under-recruitment of the VLPFC (Heatherton and Wagner, 2011). Recent work has called for modification to this prevailing paradigm, suggesting that while this conceptualization of VLPFC recruitment may be correct in the short-term (i.e. effective inhibition of distress during the aversive experience), such VLPFC recruitment undermines longer-term selfregulatory success (Chester and DeWall, 2014; Chester and Riva, 2016; Chester et al., 2016). As evidence for this new approach, performing aversive, taxing and prefrontally mediated tasks has been linked to subsequent self-regulatory failure (e.g. Inzlicht and Gutsell, 2007). Participants who were attempting to restrict their calorie intake exhibited greater reward reactivity to food stimuli and less functional connectivity between reward regions and the lateral PFC after they had to repeatedly regulate their attention away from a distracting stimulus (Wagner et al., 2013). When racially biased Whites interacted face-to-face with a Black individual, their lateral PFC recruitment to Black faces predicted subsequent self-control impairment (Richeson et al., 2003). These findings suggest that greater lateral PFC recruitment during aversive experiences may not prove adaptive in the long-term.

In the context of rejection, greater VLPFC recruitment during rejection predicted a magnified and prefrontally dysregulated ventral striatum response to appetitive cues on a subsequent lab task (Chester and DeWall, 2014). Extending outside of the lab, greater rejection-related VLPFC activity was associated with self-regulatory failures and increased cravings (Chester and DeWall, 2014). These results formed the basis of the excessive recruitment model, which posits that VLPFC recruitment in response to aversive experiences undermines subsequent 
self-regulation by impairing the VLPFC's regulatory effects on the ventral striatum (Chester and DeWall, 2014; Chester and Riva, 2016; Chester et al., 2016).

Further evidence for this model was found by observing that individuals who chronically experienced self-regulatory failure in response to aversive experiences also exhibited an exacerbated VLPFC response to aversive situations (Chester et al., 2016). This greater VLPFC response was associated with poorer inhibitory success and predicted greater alcohol consumption 1 month and 1 year later. It is important to note that selfregulation is not localized to the VLPFC, but is subserved by a host of other regions including the dorsolateral and dorsomedial PFC (Kober et al., 2010). The excessive recruitment model may indeed apply to these other regions, yet the evidence for making such claims about these other brain regions is currently lacking. As such, our hypotheses focus on the role of the VLPFC in self-regulatory failure. Because aggression can be construed as a self-regulatory failure (Denson et al., 2012), this excessive recruitment model might help explain why rejected people behave aggressively. Further, this model may help explain larger patterns of aggressive behavior that extend beyond the individual rejection incident.

\section{Trait aggression: a potential reinforcing role of striatal responses to revenge}

Physical aggression is typically thought of in the context of a single act, but aggression is also a dispositional, trait-like construct (Buss and Perry, 1992). Trait aggression shows substantial generalizability across cultures, between-individual variability, predictive validity of actual aggressive behavior and withinindividual test-retest reliability (Huesmann et al., 1984; Buss and Perry, 1992; Côté et al., 2006; Gerevich et al., 2007; Webster et al., 2014). Together, these findings provide substantial support for the existence of physical aggressiveness as a personality trait.

The underlying neurobiology of trait physical aggression remains largely unknown, with few published studies on this topic (e.g. Carré et al., 2013). An unexamined neural mechanism might underlie trait aggression: striatally mediated reinforcement. The ventral striatum's role in promoting the reinforcement of behaviors that become habitual (e.g. cocaine and alcohol abuse) is well-established (Everitt and Robbins, 2005). As a behavior that recruits the ventral striatum (Chester and DeWall, 2016), retaliatory aggression is a candidate for an act that can become striatally reinforced, leading to durable patterns of aggressive behavior across time and situations.

\section{The present study}

The main goal of this project was to better understand the neural mechanisms of the rejection-aggression link and how they might contribute to larger patterns of aggressive traits. Based off the excessive recruitment model (Chester et al., 2016), we predicted that greater VLPFC activity during social rejection would be associated with more ventral striatum activity during opportunities for retaliatory aggression. Seeking to replicate previous work (Chester and DeWall, 2016), we further predicted that ventral striatum activity during retaliatory opportunities would positively correlate with greater actual retaliation. These findings would support a temporal sequence whereby VLPFC activity during rejection promotes subsequent retaliation through a magnified ventral striatum response. We further predicted that dispositionally, physically aggressive individuals would exhibit greater dysregulation in the ability of the VLPFC to functionally inhibit the ventral striatum during retaliatory aggression.

To test these predictions, a sample of undergraduates experienced social acceptance and then rejection from two same-sex strangers and then were given an opportunity to aggressively retaliate against one of their rejecters, all while undergoing functional magnetic resonance imaging (fMRI). Participants then reported the extent of their aggressive traits and another measure of whether participants typically experienced pleasure during retaliatory aggression, which served to assist our reverse inference that the ventral striatum activity that we expected to observe during retaliatory aggression reflected reward and not some other process.

\section{Materials and methods \\ Ethics statement}

All participants provided informed consent before performing any research procedures, and all research procedures were conducted in accordance with human participants protection regulations as set forth by governmental and institutional policies. We, as authors, declare no conflicts of interest relevant to the research described in our manuscript. Data from a subset of these participants have been published in a separate manuscript (Chester and DeWall, 2018a).

\section{Participants}

Participants were 60 healthy, right-handed, English-fluent, young adults (38 females, 22 males; age: $M=20.28, S D=2.77$, range: 18-30). Participants were either undergraduates recruited through the introductory psychology subject pool in exchange for credit towards their course's research requirement and an image of their brain, or general community members recruited in exchange for $\$ 50$ and an image of their brain. Exclusionary criteria were assessed by an online questionnaire, which included: body mass index above 30, claustrophobia, color blindness, mental or neural pathology, metallic objects in the body, prior head trauma and psychoactive medication use.

\section{Materials}

Angry mood improvement inventory. The 32-item Angry Mood Improvement Inventory (AMII) assesses the degree to which individuals tend to control and express their aggressive behavior to improve their mood when they are upset (Bushman et al., 2001). The eight-item Expression-Outwards subscale of the AMII assesses the tendency to express aggression outwardly in order to experience mood repair (sample items: 'To improve my mood when I am upset, I express my anger', 'To improve my mood when I am upset, I strike out at whatever angers me'). Participants indicate the frequency of these mood-motivated actions along a 1 (Never) to 5 (Often) scale.

Brief aggression questionnaire. To measure trait physical aggression, we employed the Brief Aggression Questionnaire (BAQ; Webster et al., 2014). The BAQ contains 12 items that comprise four factors: anger (sample item: 'I have trouble controlling my temper'), hostility (sample item: 'I sometimes feel that people are laughing at me behind my back'), physical aggression (sample item: 'Given enough provocation, I may hit another person') and verbal aggression (sample item: 'My friends say that I'm 
somewhat argumentative'). Participants responded to each item along a 1 (disagree) to 7 (agree) scale.

\section{Procedure}

Participants arrived at the neuroimaging laboratory where they had the study explained to them, which entailed a cover story that the study was actually examining the role of brain functioning during various cognitive tasks in promoting alcohol misuse. Further, participants were instructed that they would be completing the study with two partners who were in nearby testing rooms. To ensure the believability of this deception, participants were told that they were the first participant to arrive and then asked to select a piece of paper that would determine which of the three MRI scanners they would be placed in (in reality, there was only one MRI scanner). After being 'assigned' to their MRI scanner, participants were then screened to ensure they would be safe and comfortable in the MRI environment, and then practiced the aggression task they would complete in the MRI scanner. Participants were then placed in an MRI scanner and had a high-resolution structural scan taken of their brain (see MRI Data Acquisition \& Preprocessing section for more details).

Cyberball task. To induce an experience of social rejection in the functional neuroimaging environment, we employed the Cyberball social rejection task (Williams et al., 2000; Eisenberger et al., 2003; Chester et al., 2014). In this task, participants were instructed to play a virtual ball-tossing game with two fictitious partners. The ostensible purpose of the task was for participants to mentally visualize the task as if it were occurring in real life, so that we might understand the neural underpinnings of the human imagination. The task proceeded across three blocks. In the first two blocks, participants received an equal number of ball-tosses from their two partners for $60 \mathrm{~s}$ per block (Acceptance condition). However, in the third block, after $30 \mathrm{~s}$, participants stopped receiving the ball from their partners who continuously threw it back-and-forth to one another for $50 \mathrm{~s}$ (Rejection condition). Baseline activation was captured by $10 \mathrm{~s}$ 'Rest' trials that preceded each of the three blocks. Total task time was $3 \mathrm{~m} 50 \mathrm{~s}$.

Aggression task. After the rejection task, participants completed an aggression task used in previous fMRI studies of aggression (Krämer et al., 2007; Chester and DeWall, 2016). In this task, participants competed against one of their fictitious Cyberball partners, who was supposedly in an MRI scanner nearby, to see who could press a button faster. As an ostensible motivational component of the task, participants were punished if they lost the competition via an aversive noise blast. Conversely, if participants won the competition their opponent heard the noise blast and they did not. Crucially, the volume of the noise blast delivered to their opponent was set by the participant and served as the measure of aggressive behavior.

The task consisted of 14 blocks, with each block containing six trials. Each block began with a 10 s fixation cross that modeled baseline neural activity. Then, participants completed a $7.5 \mathrm{~s}$ aggression trial in which they pressed a button that set the volume of their partner's noise blast. A blank screen then appeared for a jittered duration (0.5/1.0/1.5 s), which gave way to a competition trial in which participants pressed a button as fast as they could when a red square appeared on the screen (4.5/4.0/3.5 s duration). Participants then saw what volume level their opponent set for them ( $5 \mathrm{~s}$ duration). Finally, participants saw whether they won or lost the competition ( 5 s duration). If participants lost the competition, they heard an aversive noise blast that varied from 1 (silence) to 4 (extremely loud, though not dangerous). Whether a given aggression trial was preceded by their opponent setting a loud $(3,4)$ or soft $(1,2)$ volume level determined whether the given trial was retaliatory (after a loud blast) or non-retaliatory (after a soft blast). Such retaliatory and non-retaliatory trials were split fairly evenly (six retaliatory and eight non-retaliatory) and randomly presented with the exception of the first trial, which was always non-retaliatory. Wins and losses were randomized and split evenly (seven wins and seven losses). Each of the 14 blocks lasted for $32.5 \mathrm{~s}$, for a total task time of $7 \mathrm{~m} 35 \mathrm{~s}$.

Participants completed a series of other functional scans that were part of a separate project on impulsivity, and then exited the scanner. Participants were placed in a nearby testing room and completed a computerized battery of questionnaires including a demographics survey, the Brief Aggression Questionnaire, and the Angry Mood Improvement Inventory. Participants were then fully debriefed about the deception inherent in the study and escorted from the laboratory with thanks. At the onset of this debriefing was a structured suspicion probe that an experimenter verbally administered to each participant (e.g. what do you think this study was about?). No participants expressed significant suspicion about the deceptive elements of the study.

\section{MRI data acquisition and preprocessing}

All MRI data were obtained using a 3.0 tesla Siemens Magnetom Trio scanner. Echo planar BOLD images were acquired with a T2*-weighted gradient across the entire brain with a 3D shim (matrix size $=64 \times 64$, field of view $=224 \mathrm{~mm}$, echo time $=28 \mathrm{~ms}$, repetition time $=2.5 \mathrm{~s}, 3.5 \mathrm{~mm}^{3}$ isotropic voxel size, 40 interleaved axial slices, flip angle $=90^{\circ}$ ). To allow for registration to native space, a coplanar T1-weighted MP-RAGE scan was also acquired from each participant $\left(1 \mathrm{~mm}^{3}\right.$ isotropic voxel size, echo time $=2.56 \mathrm{~ms}$, repetition time $=1.69 \mathrm{~s}$, flip angle $=12^{\circ}$ ).

The Oxford Center for Functional MRI of the Brain (FMRIB)'s Software Library (FSL version 5.0) was used to conduct all preprocessing and fMRI analyses (Smith et al., 2004; Woolrich et al., 2009). Reconstructed functional volumes underwent head motion correction to the median functional volume using FSL's MCFLIRT tool. FSL's Brain Extraction Tool was used to remove non-brain tissue from all functional and structural volumes using a fractional intensity threshold of 0.5. After a series of data quality checks, functional volumes underwent interleaved slice-timing correction, pre-whitening, spatial smoothing (using a $5 \mathrm{~mm}$ full-width-half-maximum Gaussian kernel) and temporal high-pass filtering (120s cutoff). These processed brain volumes were then fed into subsequent data analyses.

\section{Statistical analyses: Functional MRI}

Preprocessed fMRI datasets from both the rejection and aggression tasks were analyzed using two levels of general linear models.

First level (within-participants). Each participant's whole-brain functional volumes were entered into a fixed-effects analysis that modeled trials as events using a canonical double-gamma hemodynamic response function with a temporal derivative. Regressors for the rejection task included Acceptance and Rejection blocks while leaving 'Rest' trials un-modeled. 'Get 
Ready' trials were modeled as a nuisance regressor. Regressorsof-interest for the aggression task included Retaliatory Aggression and Non-Retaliatory Aggression, while leaving fixation trials un-modeled. Competition, Pre-Competition, High Provocation, Low Provocation, Win and Lose trials were included as nuisance regressors. All six head motion parameters from each participant were modeled as nuisance regressors for each task.

For the rejection task, linear contrasts compared rejection to acceptance (Rejection $>$ Acceptance contrast). For the aggression task, linear contrasts compared retaliatory and nonretaliatory aggression to each other and to baseline fixation trials (Retaliatory Aggression $>$ Non-Retaliatory Aggression contrast, Retaliatory Aggression $>$ Baseline contrast, NonRetaliatory Aggression $>$ Baseline contrast). Resulting contrast images from these analyses were first linearly registered to native space structural volumes and then spatially normalized to a Montreal Neurological Institute (MNI) stereotaxic space template image (resampled into $2 \times 2 \times 2 \mathrm{~mm}^{3}$ voxels).

Second level (across-participants). Each participant's contrast volumes from the first level were then fed into FLAME 1's group level, mixed effects GLM that created group average maps for all four contrasts across the entire brain. Cluster-based thresholding was applied to each of the group activation maps (Worsley, 2001; Heller et al., 2006). Clusters were determined by applying a $Z>2.3$ threshold to the voxels of each of the group-average, whole-brain activation maps. Family-wise error correction was then applied to each cluster based on Gaussian random field theory $(P<0.05)$.

Psychophysiological interaction analysis. To assess functional connectivity during retaliatory aggression, a psychophysiological interaction (PPI) analysis was performed with the bilateral ventral striatum as a seed region-of-interest (ROI) using an anatomically and functionally defined region-of-interest (ROI) mask from the Wake Forest University Pickatlas (Maldjian et al., 2003). This took the form of a first level, within-participants analysis with the addition of two new regressors to the previously described GLM: the mean-centered timecourse of ventral striatum activity across the aggression task, and an interaction term multiplying the ventral striatum timecourse by retaliatory aggression trials. Linear contrasts compared the interaction between participants' ventral striatum timecourses and retaliatory aggression, and their interaction to participants' implicit baseline. Activation maps from this analysis were then fed into a whole-brain regression analysis in which brain activity estimates from the PPI analysis were correlated with participants' trait physical aggression levels. Clusters were determined by applying a $Z>2.3$ threshold to each of the group-average, whole-brain activation maps. Family-wise error correction was then applied to each cluster based on Gaussian random field theory $(P<0.05)$. All tests were two-tailed.

\section{Statistical analyses: mediation modeling, ROI creation and parameter estimate extraction}

In order to test whether participants' VLPFC recruitment during rejection predicted greater subsequent aggression through greater striatal activity during retaliatory aggression, we conducted a mediation analysis (using the PROCESS version 2.0 macro for SPSS, model 4, 5000 bias-corrected and accelerated resamples; Hayes, 2012). VLPFC activity was obtained from $8 \mathrm{~mm}$ spherical ROIs centered on peak activation voxels from the rejection main effect contrast. Voxels were determined to be within the VLPFC using the Automated Anatomical Labeling (AAL) atlas' opercular, orbital and triangular portions of the inferior frontal gyrus (Tzourio-Mazoyer et al., 2002). Functional data from the voxels that comprised each ROI were converted to units of percent signal change, averaged across each participant and extracted as outlined by Mumford, J. http://mumford.bol. ucla.edu/perchange_guide.pdf.

Another such mediation model was run, except that connectivity estimates from the Retaliatory Aggression PPI analysis were entered as a mediator, instead of VLPFC recruitment during rejection. This additional, exploratory mediation analysis served the purpose of examining whether striatum-based functional connectivity helped to explain the link between VLPFC recruitment during rejection and subsequent retaliatory aggression. All tests were two-tailed.

\section{Open practices}

De-identified data necessary to reproduce all analyses from this project have been made publicly available at https://osf.io/ n5bwh/files/.

\section{Results}

\section{Descriptive statistics}

BAQ. One participant failed to complete the Brief Aggression Questionnaire. Analyses were constrained to the Physical Aggression subscale of this questionnaire, as the aggressive behavior measured by our MRI task was physical, as opposed to verbal, in nature. Physical Aggression subscale scores exhibited substantial variability across the scale's possible 1-7 range, $\mathrm{M}=2.92, \quad \mathrm{SD}=1.70$, observed range $=1.00-7.00$, Cronbach's $\alpha=0.84$.

AMII. One participant failed to complete the Angry Mood Improvement Inventory. We calculated Express-Outwards, $\alpha=0.74$, Express-Inwards, $\alpha=0.81$, Control-Outwards, $\alpha=0.84$ and Control-Inwards, $\alpha=0.77$, subscale scores by averaging across each participant's corresponding responses. ExpressOutwards subscale scores exhibited substantial variability across the scale's possible $1-5$ range, $M=2.19, S D=0.49$, observed range $=1.38-3.38$ and were positive correlated with trait physical aggression from the BAQ $r(57)=0.38, P=0.003$.

Aggression task. Volume settings were internally consistent, $\alpha=.91$, and thus averaged across all 14 trials of the aggression task, as well as the six retaliatory trials, $\alpha=.81$, and eight nonretaliatory trials, $\alpha=.85$, to create three aggression scores (i.e. total, retaliatory and non-retaliatory) for each participant, possible range of 1-4. Validating our within-subjects provocation manipulation, participants selected louder noise blasts after high provocation (i.e. retaliatory aggression: $M=2.58, S D=0.87$, observed range $=1.00-4.00$ ), than after low provocation (i.e. non-retaliatory aggression: $M=2.41, \quad S D=0.79$, observed range $=1.00-4.00), \quad t(59)=2.96, \quad P=0.004$, Cohen's $d_{\text {dependent }-}$ means $=0.41$.

\section{Neural correlates of rejection}

Social rejection (compared to social acceptance) was both positively and negatively associated with large swaths of neural activity (see Tables 1 and 2). The positively associated regions 
Table 1. Brain regions positively associated with Reject $>$ Accept during Cyberball

\begin{tabular}{|c|c|c|c|c|}
\hline Cluster & Voxels & Brain region & Peak Z & Peak $x, y, z$ \\
\hline \multirow[t]{6}{*}{1} & 25205 & VLPFC/anterior insula & 6.07 & $48,22,12$ \\
\hline & & & 5.98 & $52,22,12$ \\
\hline & & & 5.88 & $46,36,-6$ \\
\hline & & & 5.81 & $56,26,12$ \\
\hline & & Temporoparietal junction & 5.77 & $50,-44,20$ \\
\hline & & $\begin{array}{l}\text { Middle temporal gyrus/ } \\
\text { temporal pole }\end{array}$ & 5.68 & $56,-10,-16$ \\
\hline \multirow[t]{6}{*}{2} & 10661 & VLPFC & 6.46 & $-42,20,-26$ \\
\hline & & Temporoparietal junction & 6.00 & $-46,-66,22$ \\
\hline & & $\begin{array}{l}\text { Middle temporal gyrus/ } \\
\text { temporal pole }\end{array}$ & 5.89 & $-46,8,-26$ \\
\hline & & Temporoparietal junction & 5.72 & $-50,-66,18$ \\
\hline & & & 5.65 & $-44,-70,24$ \\
\hline & & Anterior insula & 5.61 & $-36,24,-6$ \\
\hline \multirow[t]{3}{*}{3} & 9239 & Dorsomedial PFC & 7.52 & $10,52,36$ \\
\hline & & & 7.09 & $8,46,30$ \\
\hline & & & 7.01 & $6,46,26$ \\
\hline 4 & 1492 & Posterior cingulate cortex & 5.49 & $4,-54,34$ \\
\hline 5 & 1382 & Thalamus/caudate & 5.34 & $6,-10,10$ \\
\hline 6 & 1218 & Occipital cortex & 5.03 & $-12,-94,4$ \\
\hline 7 & 599 & Brainstem & 3.53 & $4,-30,-30$ \\
\hline
\end{tabular}

Notes and Sources: Each cluster is displayed with rows for all local maxima.

included anticipated brain areas previously associated with social pain (bilateral anterior insula, thalamus) and social pain regulation (VLPFC; Eisenberger, 2012; Figure 1). Additional activity was observed in what is popularly known as the mentalizing network [dorsomedial prefrontal cortex (DMPFC), posterior cingulate cortex\precuneus (PCC), bilateral temporoparietal junction (TPJ) and bilateral temporal pole (TP); Figure 1; Frith and Frith (2006)].

Within the VLPFC ROI, three local maxima were observed. Three VLPFC ROI masks were constructed by creating $8 \mathrm{~mm}$ binary spheres around each local maximum (Figure 2). Parameter estimates from this rejection contrast were extracted, in the form of percent BOLD signal change, from each of these three VLPFC ROIs for use in later correlational and mediation analyses.

\section{Neural correlates of retaliatory aggression}

Across the entire brain, retaliatory aggression (as compared to non-retaliatory aggression) was positively associated with activity in the right [ 952 voxels; peak voxel: $Z=3.68$, MNI coordinates $(x, y, z): 34,-14,24]$ and left (688 voxels; peak voxel: $Z=4.62$, coordinates: $-50,-20,8)$ posterior insula and negatively associated with activity in the occipital lobe (2101 voxels; peak voxel: $Z=-4.92$, coordinates: $38,-88,-6)$. Parameter estimates from this retaliatory aggression contrast, in the form of percent signal change, were extracted from the bilateral ventral striatum for use in later correlational and mediation analyses. Replicating previous research (Chester and DeWall, 2016), retaliatory aggression related activity in the ventral striatum was associated with higher scores on the Expression-Outwards subscale of the Angry Mood Improvement Inventory (AMII), $r(57)=0.33, P=0.010$, even after using multiple linear regression to control for the other three AMII subscales, $\beta=0.30$, $t(54)=2.18, P=0.034$.
Table 2. Brain regions negatively associated with Reject $>$ Accept during Cyberball

\begin{tabular}{|c|c|c|c|c|}
\hline Cluster & Voxels & Brain region & Peak Z & Peak $x, y, z$ \\
\hline \multirow[t]{6}{*}{1} & \multirow[t]{6}{*}{7616} & Posterior insula & -6.54 & $-38,-6,16$ \\
\hline & & \multirow[t]{2}{*}{ Precentral gyrus } & -5.69 & $-30,-14,64$ \\
\hline & & & -5.33 & $-36,-16,66$ \\
\hline & & Supplemental motor area & -5.26 & $-4,-6,54$ \\
\hline & & \multirow[t]{2}{*}{ Postcentral gyrus } & -5.14 & $-48,-34,56$ \\
\hline & & & -4.98 & $-52,-28,50$ \\
\hline \multirow[t]{6}{*}{2} & \multirow[t]{6}{*}{834} & \multirow[t]{6}{*}{ Dorsolateral PFC } & -4.29 & $-40,44,8$ \\
\hline & & & -3.67 & $-46,28,28$ \\
\hline & & & -3.60 & $-34,32,28$ \\
\hline & & & -3.58 & $-34,24,24$ \\
\hline & & & -3.46 & $-40,34,34$ \\
\hline & & & -3.29 & $-38,52,16$ \\
\hline \multirow[t]{6}{*}{3} & \multirow[t]{6}{*}{478} & Superior parietal lobule & -3.77 & $30,-48,68$ \\
\hline & & Supramarginal gyrus & -3.56 & $48,-36,54$ \\
\hline & & Postcentral gyrus & -3.51 & $42,-28,40$ \\
\hline & & Superior parietal lobule & -3.46 & $36,-46,64$ \\
\hline & & Postcentral gyrus & -3.09 & $46,-32,50$ \\
\hline & & Superior parietal lobule & -3.02 & $32,-54,64$ \\
\hline
\end{tabular}

Notes and Sources: Each cluster is displayed with rows for all local maxima.

Crucially, the neuroimaging results from the Retaliatory Aggression > Non-Retaliatory Aggression contrast only reflect the opportunity to engage in retaliatory aggression and do not reflect the actual neural correlates of retaliatory aggressive behavior itself. We regressed participants' retaliatory aggression scores onto whole-brain neural activity from the Retaliatory Aggression $>$ Non-Retaliatory Aggression contrast to identify the true neural correlates of retaliatory aggression. Whole-brain regression analyses revealed no significant correlates of retaliatory aggressive behavior. However, when analyses were constrained to the bilateral ventral striatum using an ROI approach, we observed significant positive associations with the left (9 voxels; peak voxel: $Z=2.88$, coordinates $=-12,4,-10$ ) and right (10 voxels; peak voxel: $Z=2.63$, coordinates $=14,6,-12$ ) ventral striatum (Figure 3).

\section{Frontostriatal associations with retaliatory aggression}

Of the three spherical VLPFC ROIs, rejection-related activity in the most rostral of the ROIs was positively associated with bilateral ventral striatum activity during retaliatory aggression (Retaliatory Aggression $>$ Non-Retaliatory Aggression contrast), $r(58)=0.346, P=0.007$. Bilateral ventral striatum activity during retaliatory aggression was associated with greater retaliatory aggression, $r(58)=0.342, P=0.007$. This pattern of correlations suggested the presence of an indirect effect.

To test this potential indirect effect, bilateral ventral striatum activity during retaliatory aggression was modeled as a mediator of the effect of VLPFC activity during rejection on subsequent retaliatory aggressive behavior. Based off the previously significant association with aggression-related activity in the ventral striatum, parameter estimates from the most rostral VLPFC ROI was modeled as the independent variable. A significant indirect effect was observed from this model, $B=1.41$, $\mathrm{SE}=0.70,95 \% \mathrm{CI}=0.30,3.22$. This overall model explained $11.90 \%$ of the variance in retaliatory aggression, $F(2,57)=3.85$, $P=0.027$ (Figure 4). 

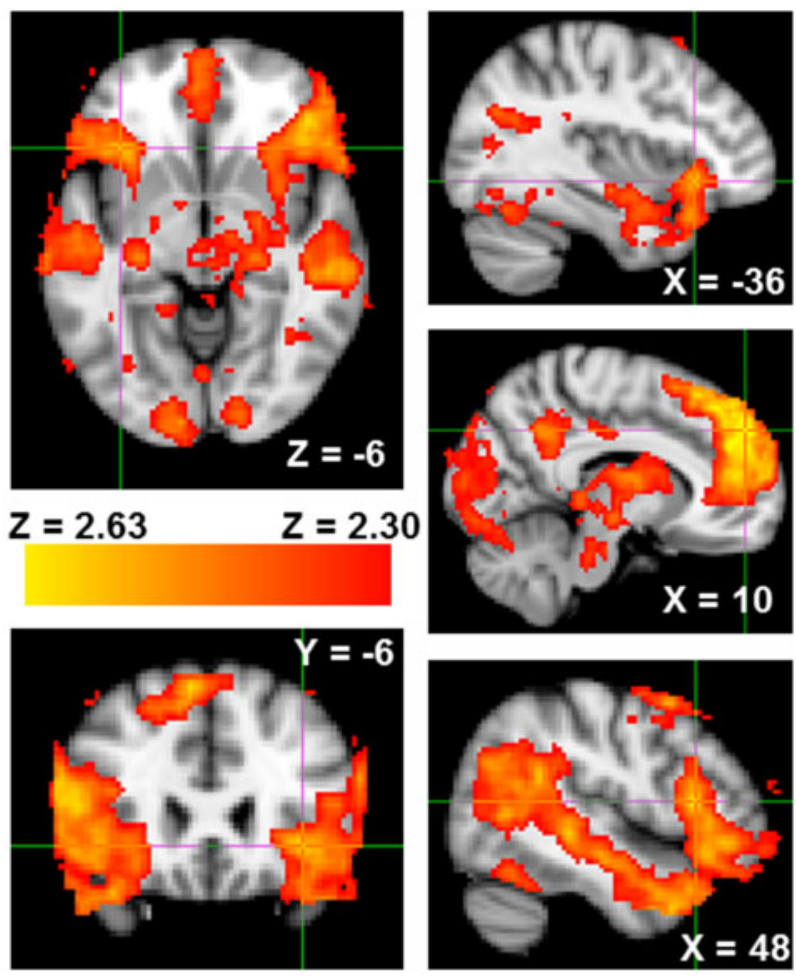

Fig. 1. Greater neural activity from the Reject $>$ Accept contrast of the Cyberball task in bilateral anterior insula, VLPFC and mentalizing network. Coordinates are in MNI space.

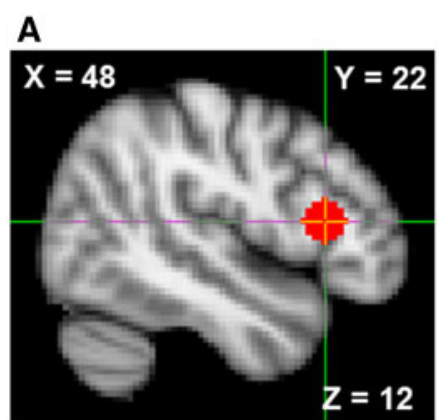

B

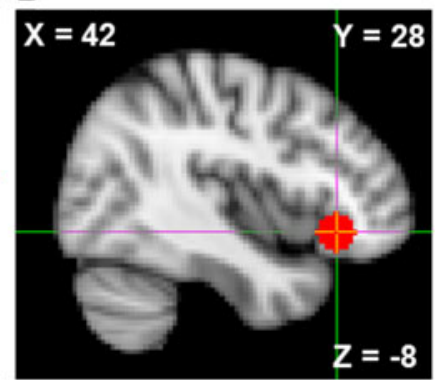

D
C

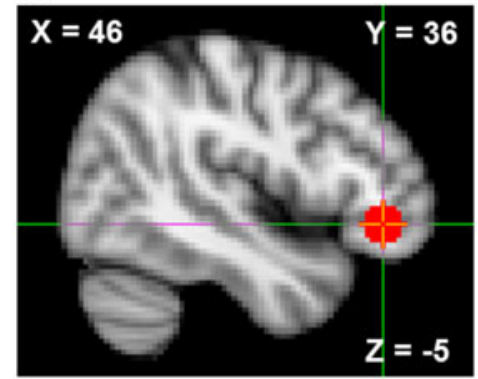

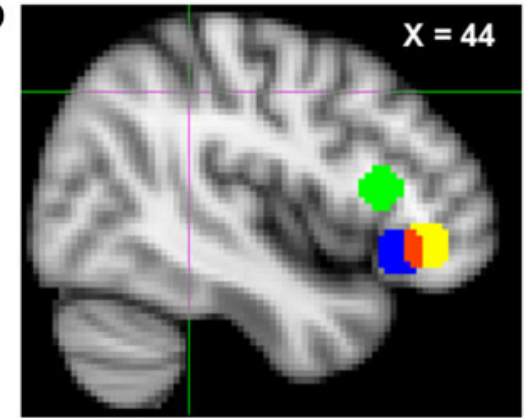

Fig. 2. (A-C) Spherical VLPFC ROIs constructed by centering each sphere on one of three local maxima from the Reject $>$ Accept contrast. (D) All three ROIs displayed simultaneously, red voxels display overlap between ROIs B and C. Coordinates are in MNI space.

Does VLPFC activity simply reflect stronger affective responses to rejection?

An alternative account for our findings could be that the VLPFC activity we observed during social rejection might simply reflect a greater affective response to this event, and not the regulatory account we have proposed. If this alternative explanation is correct, then we should be able to replicate the positive associations that were observed between VLPFC activity (during rejection) and ventral striatum activity (during aggression) with other neural regions that subserve social pain (i.e. the DACC and anterior insula; Eisenberger, 2012).To test whether this was 


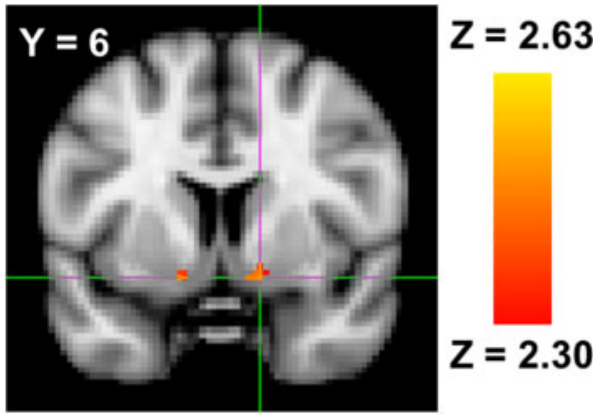

Fig. 3. Bilateral ventral striatum activity associated with greater retaliatory aggressive behavior on the aggression task.

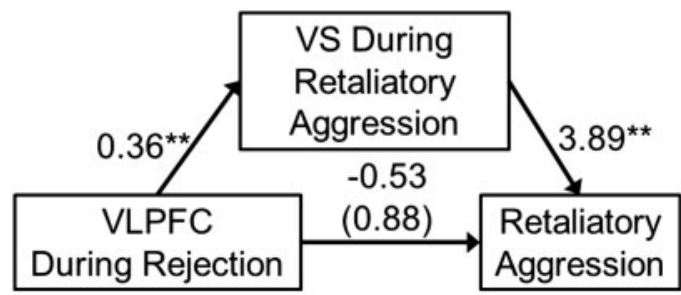

Fig. 4. An indirect effect whereby ventrolateral prefrontal cortex (VLPFC) activity during rejection was associated with greater retaliatory aggression through greater bilateral ventral striatum (VS) activity during such retaliatory aggression. Values represent unstandardized regression coefficients and the value in parentheses represent the direct effect (i.e. the total effect minus the indirect effect). ${ }^{* *} \mathrm{P}<0.01$.

the case, we extracted rejection-related activation from the DACC and bilateral anterior insula (for DACC mask see Chester et al., 2015; anterior insula mask see Chester et al., 2014) and correlated them with retaliatory-aggression-related activity in the bilateral ventral striatum. Failing to support this alternative account, VLPFC activity was unassociated with DACC, $r(58)=-0.12, P=0.378$, or anterior insula, $r(58)=-0.01, P=0.929$, activity during rejection.

\section{Dispositional aggression and frontostriatal connectivity during retaliatory aggression}

Dispositional physical aggression was positively associated with retaliatory aggression, $r(57)=0.29, P=0.029$, though not with greater ventral striatum activity during retaliatory aggression, $r(57)=0.10, P=0.435$. Subsequent analyses tested whether trait physical aggression was associated with altered functional connectivity between cortical brain regions and the bilateral ventral striatum, during retaliatory aggressive behavior. The combined psychophysiological interaction (PPI) and whole-brain regression analyses revealed a single, negatively correlated cluster in the right VLPFC (peak voxel: $Z=-4.21$, $P=0.004$, MNI coordinates $[x, y, z]=34,52,6 ; 529$ contiguous voxels; Figure 5; Brodmann's Areas 46 and 47), though some voxels extended into the rostral (Brodmann's Area 10) and ventral (Brodmann's Area 11) medial PFC.

In a post hoc, exploratory fashion, functional connectivity estimates from the VLPFC cluster observed in the Retaliatory Aggression PPI analysis were entered as a mediator into a mediation analysis (the size of these connectivity units were so small that we artificially inflated them by multiplying them times 100 for this analysis, so that effect size estimates would be interpretable). Retaliatory aggression was modeled as the dependent

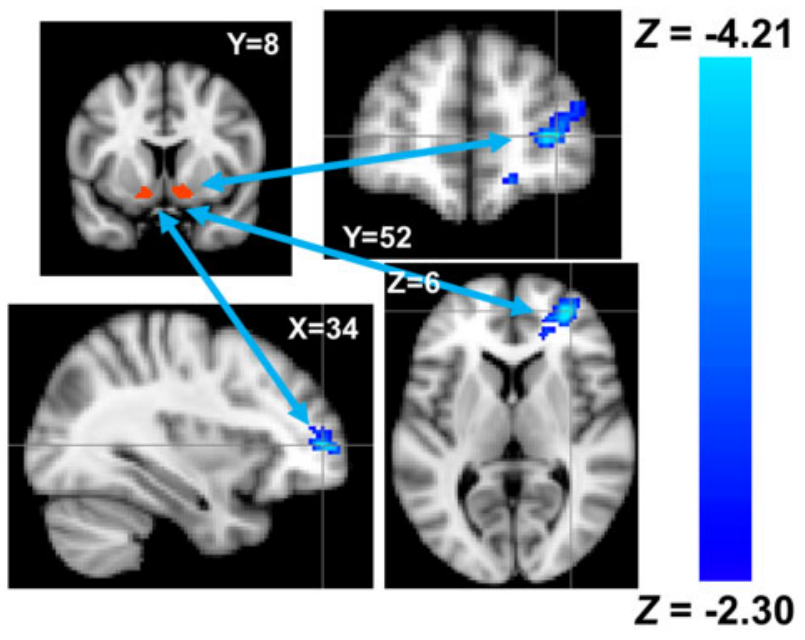

Fig. 5. Reduced functional connectivity between the seed region in the ventral striatum (red) and the ventrolateral prefrontal cortex (blue) among individuals higher in trait physical aggression. Coordinates are in MNI space.

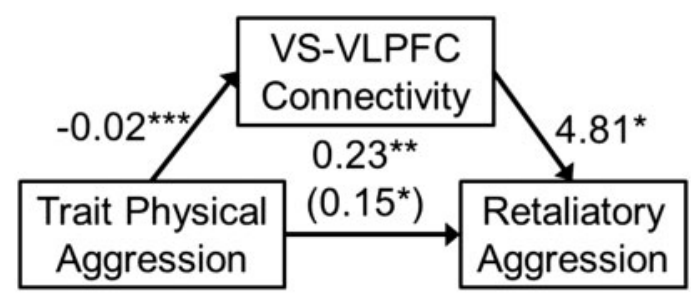

Fig. 6. An indirect effect whereby dispositional physical aggressiveness was associated with greater aggression through less functional connectivity between the ventral striatum (VS) and ventrolateral prefrontal cortex (VLPFC) during such retaliatory aggression. Values represent unstandardized regression coefficients and the value in parentheses represent the direct effect. ${ }^{*} \mathrm{P}<0.05$, ${ }^{* *} \mathrm{P}<0.01,{ }^{* * *} \mathrm{P}<0.001$.

variable and trait physical aggression was modeled as the independent variable. A significant indirect effect was observed from this model, $B=-0.08, S E=0.04,95 \% C I=-0.18,-0.01$, whereby trait physical aggression predicted more retaliatory aggression through reduced functional connectivity between the ventral striatum and VLPFC during retaliatory aggression. This overall model explained $17.17 \%$ of the variance in retaliatory aggression, $F(2,56)=5.80, P=0.005$ (Figure 6). As an indicator of statistical suppression, the direct effect of trait physical aggression on retaliatory aggression became stronger after controlling for the indirect effect of frontostriatal connectivity (Figure 6; Mackinnon et al., 2000).

\section{Discussion}

Why do rejected people behave aggressively? Although rejection reliably increases aggression, it remains unclear what neurological mechanisms help explain this relationship. Our investigation fills this gap in the literature by offering a comprehensive account of the neural correlates of rejection-related aggression. Building off of the excessive recruitment model (Chester and DeWall, 2014; Chester et al., 2016), we examined whether taxing the brain's regulatory functions during rejection would promote subsequent retaliatory aggression by unleashing neural reward circuitry, which would motivate retaliation by rendering such revenge more enticing. 


\section{Implications for the neuroscience of social rejection}

We replicated previous research demonstrating that social rejection, compared to acceptance, is associated with greater activity in brain networks that subserve social pain (anterior insula; Eisenberger et al., 2003), social pain regulation (VLPFC; Chester and DeWall, 2014) and mentalizing about others' mind state (Falk et al., 2014). These replications support Social Pain Overlap Theory's central tenet that rejection is a markedly painful experience that recruits the affective, and less often somatic, aspects of the brain's pain circuitry (Eisenberger and Lieberman, 2004; Eisenberger, 2012). Notably absent from our observed neural correlates of rejection was the DACC, which is typically observed in experiences of social rejection (Kawamoto et al., 2012). It remains uncertain why this region was not reactive to rejection in our experiment.

\section{Implications for the neuroscience of retaliatory aggression}

Opportunities to retaliate against one of the participants' rejecters were associated with greater activity in the posterior insula, which replicates previous work on retaliatory aggression (Chester and DeWall, 2016; Emmerling et al., 2016). The posterior insula's role in somatic and visceral interoception (Craig, 2011) may indicate that retaliatory aggression is experienced as a physically grounded state or signify an alertness to somatic cues. When actual retaliatory aggressive behavior was correlated with these retaliatory brain activity estimates, we observed significant, positive associations in the bilateral ventral striatum (Chester and DeWall, 2016). The added sensitivity gained from using the whole-brain regression approach suggests that this approach may be useful to researchers who regularly model the types of trials employed in their tasks as explanatory variables, but less commonly model participants' actual responses to those task features.

Adopting an ROI approach, we also replicated the association between activity in the ventral striatum during retaliatory aggression and the extent of actual retaliation that participants inflicted on their opponents (Chester and DeWall, 2016). Further, we replicated the correlation between such striatal activity and participants' self-reported tendencies to react aggressively in order to improve their mood (as in Chester and DeWall, 2016). This replication lends confidence to our striatum-reward reverse inference, which is further supported by the extensive evidence for the ventral striatum's involvement in reward (Burgdorf and Panksepp, 2006; Ikemoto, 2007; Sabatinelli et al., 2007; Kringelbach and Berridge, 2009; Berridge and Kringelbach, 2013). A reliably observed striatal response to retaliatory aggression supports the growing literature implicating positive affect, pleasure and reward and central psychological components of retaliatory aggression (Chester, 2017; Chester and DeWall, 2018b).

\section{Neurochemical nuances of reward}

However, 'reward' is a heterogeneous construct and these various sub-processes are subserved by distinct neurobiological pathways that fMRI cannot currently estimate (Berridge and Robinson, 2003). Indeed, 'liking' or pleasure is likely to be mediated by opioid transmitter pathways and spiny neurons in the shell of the ventral striatum, whereas motivational components of 'wanting' is likely to be mediated by dopaminergic transmitter pathways in this same region (Kringelbach and Berridge, 2009). Imaging and pharmacological techniques that measure and manipulate neural dopamine and opioid levels and binding sites are needed to better investigate whether 'wanting' or 'liking' is at play in retaliatory aggression. Disentangling these experiences is crucial for a comprehensive understanding of aggression's phenomenological qualities.

\section{Support for the excessive recruitment model: sometimes less is more}

In support of the excessive recruitment model of self-regulatory failure (Chester and DeWall, 2014; Chester et al., 2016), anterior VLPFC recruitment during rejection was associated with greater ventral striatum activation during retaliatory aggression. The VLPFC-striatum circuit's role in successful self-regulation is supported by these findings and further suggests that retaliatory aggression is a rewarding activity, as similar findings are observed in other rewarding behaviors such as unhealthy eating (Wagner et al., 2013). Crucially, only the most anterior ROI in our rejection-related VLPFC cluster was associated with striatal activity during aggression. The specificity of our findings to the anterior VLPFC can be interpreted in the light of research demonstrating that behavioral inhibition is localized to posterior VLPFC subregions and emotional and cognitive inhibition is localized to the anterior VLPFC (Aron et al., 2014; Buhle et al., 2014). As such, we can potentially infer that the VLPFC's regulation of social pain and not the aggressive motor act itself is what predicted subsequent increases in retaliation-related striatal activity. Further, the null association between VLPFC activity during rejection and activity in the DACC and anterior insula undermine the potential alternative account that our VLPFC activation did not reflect self-regulation, but rather more intense affective responses to rejection.

These findings cast doubt on 'the more lateral PFC, the better' approaches to the neuroscience of self-regulation and support the excessive recruitment model (Chester and DeWall, 2014; Chester et al., 2016). However, it may be that the excessive recruitment model only holds for situations characterized by negative affect (e.g. rejection), as other research has found that greater VLPFC recruitment to self-regulatory challenges outside of this affect context are predictive of self-regulatory success, not failure (Lopez et al., 2014). Further, chronic recruitment of brain regions can increases their plasticity and baseline activity (e.g. Teneback et al., 1999). If this was the case, then individuals who show exacerbated recruitment of the VLPFC during each aversive event would eventually trait this brain region to become more flexible and robust, promoting better emotionregulation. Future work is needed to understand how some forms of cortical recruitment can lead to adaptive or maladaptive changes in behavior.

\section{A reinforcement model of trait aggression}

Dispositionally and physically aggressive individuals exhibited less functional connectivity between the VLPFC and ventral striatum during retaliatory aggression. Further, this connectivity exerted a suppressing effect on their aggression, suggesting that without such neural control over reward circuitry, those high in aggressive traits would act much more aggressively than they typically do.

Such dysregulated reward activity during retaliatory belligerence might explain how aggressive traits are developed and maintained. Classic models of aggression emphasize distinct social-cognitive and affective-learning mechanisms as the process through which some individuals develop to be more 
aggressive than others (e.g. the General Aggression Model; Anderson and Bushman, 2002). This project argues for a more integrated conceptualization of affective and cognitive processes in these models, in which cognitive control mechanisms and affective impulses dynamically interact to reinforce aggressive behavior. These results also suggest avenues for future research to investigate how striatally mediated reinforcement processes can be altered to reduce the occurrence of physically aggressive dispositions. However, it remains unclear if the VLPFC connectivity with the striatum represents inhibition of the affective reward experience or a more general and behavioral inhibition of aggressive impulses. The anterior nature of this VLPFC cluster does suggest that the inhibition targeted affective and not the behavioral processes, yet more work is needed to be certain.

\section{A role for appraisal processes?}

Emotional responses to situations are determined by the appraisals of those situations (Siemer et al., 2007; Brosch et al., 2010). Further, such emotional appraisals can fundamentally alter the neural responses to emotional stimuli (Brosch and Sander, 2013; Buhle et al., 2014). Emotional responses to social rejection and aggression are no exception. For instance, externalizing responses to rejection are contingent upon the appraisal of the event (Sandstrom et al., 2003). The present research failed to include measures or manipulations of appraisal processes. However, establishing the role of subjective appraisals, beyond the objective experimental manipulations we employed, is critical to understanding the neural mechanisms of the rejection-aggression link.

\section{Limitations and future directions}

Our findings must be considered in light of the sample, which consisted of 60 undergraduates, and pose some concerns for the generalizability of our findings and the extent to which our analyses were sufficiently powered. The fact that most of our findings were close replications of previous studies, which we linked together in the same sample, provide some confidence that our results were not merely due to sampling error. An additional concern is that all findings were correlational in nature. Future work that experimentally modulates these neural systems, such as brain stimulation and pharmacological techniques, can circumvent this issue. The reverse inferences that we made are also limitations as the neural activity we observed may not actually be reflective of the psychological processes we interpreted them to signify (Poldrack, 2006). Our striatumreward associations were supported by self-report evidence, but more work is needed to ensure the fidelity of our reverse inferences. Further, the association between retaliatory aggression and ventral striatum activity (displayed in Figure 3) was observed in a relatively small number of voxels (i.e. 19), which potentially undermines the reliability of these findings. However, voxels from the entire anatomical region were used in all other analyses, which provides more inferential confidence than just using the significant voxels form the regression analysis.

Each of our aggression-related neural activity estimates may have been biased by the interleaved baseline fixation screens that we used, which may have been influenced by residual reactivity to previous elements of the task. Future work may benefit from using baseline estimates that are acquired before the aggression task. Finally, the ways in which striatal activity motivates aggression are unable to be articulated given our data. It may be that the anticipation of the striatal reward associated with aggression prospectively motivates such behavior. Conversely, it may be that the reward experienced during the aggressive act motivates more severe acts of aggression, in order to magnify the ongoing hedonic experience. Research that is able to disentangle the anticipated us in-the-moment motivational capabilities of aggression's rewarding qualities is a necessary future endeavor. Our rejection block was also confounded with the duration of the Cyberball task, which may have produced spurious patterns of brain activity, such as those we observed in the visual and auditory cortices. Finally, readers should use caution when interpreting our findings because we did not correct for the multiple comparisons that were made across the various VLPFC regions-of-interest.

\section{Conclusions}

Does the pain of rejection promote the sweetness of revenge? Our findings suggest that the answer to this question is yes, albeit indirectly. People are motivated to maintain their social connections, but also to avoid having those connections inflict excessive costs upon them (McCullough et al., 2013). Pain and pleasure are two proximate mechanisms that evolution may have co-opted to motivate individuals to avoid rejection (i.e. social pain) and to seek retribution against those who harm them (i.e. aggressive pleasure). By understanding how coping with the pain of rejection impacts our self-regulatory abilities, and how these regulatory changes render retaliation an appetitive option, we may better understand how to prevent the aggressive dividends that rejection often yields.

\section{Funding}

This work was supported by the National Institute on Drug Abuse (award \# DA05312; Lynam, Milich and DeWall), the Foundation for Personality and Social Psychology's Heritage Initiative (Chester), and the Robert S. Lipman Research Fund for the Prevention of Drug and Alcohol Abuse (Chester).

Conflict of interest. None declared.

\section{References}

Anderson, C.A., Bushman, B.J. (2002). Human aggression. Annual Review of Psychology, 53(1), 27-51.

Aron, A.R., Robbins, T.W., Poldrack, R.A. (2014). Inhibition and the right inferior frontal cortex: one decade on. Trends in Cognitive Sciences, 18(4), 177-85.

Berridge, K.C., Kringelbach, M.L. (2013). Neuroscience of affect: brain mechanisms of pleasure and displeasure. Current Opinion in Neurobiology, 23(3), 294-303.

Berridge, K.C., Robinson, T.E. (2003). Parsing reward. Trends in Neurosciences, 26(9), 507-13.

Brosch, T., Pourtois, G., Sander, D. (2010). The perception and categorisation of emotional stimuli: a review. Cognition and Emotion, 24(3), 377-400.

Brosch, T., Sander, D. (2013). Comment: the appraising brain: towards a neuro-cognitive model of appraisal processes in emotion. Emotion Review, 5(2), 163-8.

Buades-Rotger, M., Brunnlieb, C., Münte, T.F., Heldmann, M., Krämer, U.M. (2016). Winning is not enough: ventral striatum connectivity during physical aggression. Brain Imaging and Behavior, 10(1), 105-14. 
Buckley, K.E., Winkel, R.E., Leary, M.R. (2004). Reactions to acceptance and rejection: effects of level and sequence of relational evaluation. Journal of Experimental Social Psychology, 40(1), 14-28.

Buhle, J.T., Silvers, J.A., Wager, T.D., et al. (2014). Cognitive reappraisal of emotion: a meta-analysis of human neuroimaging studies. Cerebral Cortex, 24(11), 2981-90.

Burgdorf, J., Panksepp, J. (2006). The neurobiology of positive emotions. Neuroscience and Biobehavioral Reviews, 30(2), 173-87.

Bushman, B.J., Baumeister, R.F., Phillips, C.M. (2001). Do people aggress to improve their mood? Catharsis beliefs, affect regulation opportunity, and aggressive responding. Journal of Personality and Social Psychology, 81(1), 17-32.

Buss, A.H., Perry, M. (1992). The aggression questionnaire. Journal of Personality and Social Psychology, 63(3), 452-9.

Carré, J.M., Murphy, K.R., Hariri, A.R. (2013). What lies beneath the face of aggression?. Social Cognitive and Affective Neuroscience, 8(2), 224-9.

Chester, D.S. (2017). The role of positive affect in aggression. Current Directions in Psychological Science, 26(4), 366-70.

Chester, D.S., DeWall, C.N. (2014). Prefrontal recruitment during social rejection predicts greater subsequent self-regulatory imbalance and impairment: neural and longitudinal evidence. NeuroImage, 101(1), 485-93.

Chester, D.S., DeWall, C.N. (2016). The pleasure of revenge: retaliatory aggression arises from a neural imbalance toward reward. Social Cognitive and Affective Neuroscience, 11(7), 1173-82.

Chester, D.S., DeWall, C.N. (2018a). Aggression is associated with greater subsequent alcohol consumption: Shared neural basis in the ventral striatum. Aggressive Behavior, 44(3), 285-93.

Chester, D.S., DeWall, C.N. (2018b). Personality correlates of revenge-seeking: Multidimensional links to physical aggression, impulsivity, and aggressive pleasure. Aggressive Behavior, 44(3), 235-45.

Chester, D.S., Eisenberger, N.I., Pond, R.S., Richman, S.B., Bushman, B.J., DeWall, C.N. (2014). The interactive effect of social pain and executive functioning on aggression: an fMRI experiment. Social Cognitive and Affective Neuroscience, 9(5), 699-704.

Chester, D.S., Lynam, D.R., Milich, R., Powell, D.K., Andersen, A.H., DeWall, C.N. (2016). How do negative emotions impair self-control? A neural model of negative urgency. NeuroImage, 132(1), 43-50.

Chester, D.S., Pond, R.S., DeWall, C.N. (2015). Alexithymia is associated with blunted anterior cingulate response to social rejection: implications for daily rejection. Social Cognitive and Affective Neuroscience, 10(4), 517-22.

Chester, D.S., Riva, P. (2016). Brain mechanisms to regulate negative reactions to social exclusion. In: Riva, P., Eck J., editors. Social Exclusion: Psychological Approaches to Understanding and Reducing Its Impact, Cham, Switzerland: Springer International Publishing.

Chow, R.M., Tiedens, L.Z., Govan, C.L. (2008). Excluded emotions: the role of anger in antisocial responses to ostracism. Journal of Experimental Social Psychology, 44(3), 896-903.

Côté, S., Vaillancourt, T., LeBlanc, J.C., Nagin, D.S., Tremblay, R.E. (2006). The development of physical aggression from toddlerhood to pre-adolescence: a nation wide longitudinal study of Canadian children. Journal of Abnormal Child Psychology, 34(1), 68-82.

Craig, A.D. (2011). Significance of the insula for the evolution of human awareness of feelings from the body. Annals of the New York Academy of Sciences, 1225(1), 72-82.
Dambacher, F., Sack, A.T., Lobbestael, J., Arntz, A., Brugman, S., Schuhmann, T. (2014). Out of control: evidence for anterior insula involvement in motor impulsivity and reactive aggression. Social Cognitive and Affective Neuroscience, 10(4), 508-16.

Denson, T.F., DeWall, C.N., Finkel, E.J. (2012). Self-control and aggression. Current Directions in Psychological Science, 21(1), 20-5.

DeWall, C.N., Twenge, J.M., Gitter, S.A., Baumeister, R.F. (2009). It's the thought that counts: the role of hostile cognition in shaping aggressive responses to social exclusion. Journal of Personality and Social Psychology, 96(1), 45-59.

Eisenberger, N.I. (2012). The pain of social disconnection: examining the shared neural underpinnings of physical and social pain. Nature Reviews Neuroscience, 13(6), 421-34.

Eisenberger, N.I., Lieberman, M.D. (2004). Why rejection hurts: a common neural alarm system for physical and social pain. Trends in Cognitive Sciences, 8(7), 294-300.

Eisenberger, N.I., Lieberman, M.D., Williams, K.D. (2003). Does rejection hurt? An fMRI study of social exclusion. Science, 302(5643), 290-2.

Emmerling, F., Schuhmann, T., Lobbestael, J., Arntz, A., Brugman, S., Sack, A.T. (2016). The role of the insular cortex in retaliation. PLoS One, 11(4), e0152000.

Everitt, B.J., Robbins, T.W. (2005). Neural systems of reinforcement for drug addiction: from actions to habits to compulsion. Nature Neuroscience, 8(11), 1481-9.

Falk, E.B., Cascio, C.N., O'Donnell, M.B., et al. (2014). Neural responses to exclusion predict susceptibility to social influence. Journal of Adolescent Health, 54(5), S22-31.

Frith, C.D., Frith, U. (2006). The neural basis of mentalizing. Neuron, 50(4), 531-4.

Gerevich, J., Bácskai, E., Czobor, P. (2007). The generalizability of the Buss-Perry Aggression Questionnaire. International Journal of Methods in Psychiatric Research, 16(3), 124-36.

Hayes, A.F. (2012). PROCESS: a versatile computational tool for observed variable mediation, moderation, and conditional process modeling (Version 2.0) [Software]. Available: http:// www.afhayes.com/public/process2012.pdf [August 18, 2017].

Heatherton, T.F., Wagner, D.D. (2011). Cognitive neuroscience of self-regulation failure. Trends in Cognitive Sciences, 15(3), 132-9.

Heller, R., Stanley, D., Yekutieli, D., Rubin, N., Benjamini, Y. (2006). Cluster-based analysis of fMRI data. NeuroImage, 33(2), 599-608.

Huesmann, L.R., Eron, L.D., Lefkowitz, M.M., Walder, L.O. (1984). Stability of aggression over time and generations. Developmental Psychology, 20(6), 1120-34.

Ikemoto, S. (2007). Dopamine reward circuitry: two projection systems from the ventral midbrain to the nucleus accumbens-olfactory tubercle complex. Brain Research Reviews, 56(1), 27-78.

Inzlicht, M., Gutsell, J.N. (2007). Running on empty: neural signals for self-control failure. Psychological Science, 18(11), 933-7.

Kawamoto, T., Onoda, K., Nakashima, K.I., Nittono, H., Yamaguchi, S., Ura, M. (2012). Is dorsal anterior cingulate cortex activation in response to social exclusion due to expectancy violation? An fMRI study. Frontiers in Evolutionary Neuroscience, 4, 11.

Kober, H., Mende-Siedlecki, P., Kross, E.F., et al. (2010). Prefrontal-striatal pathway underlies cognitive regulation of craving. Proceedings of the National Academy of Sciences, 107(33), 14811-6.

Krämer, U.M., Jansma, H., Tempelmann, C., Münte, T.F. (2007). Tit-for-tat: the neural basis of reactive aggression. NeuroImage, 38(1), 203-11. 
Kringelbach, M.L., Berridge, K.C. (2009). Towards a functional neuroanatomy of pleasure and happiness. Trends in Cognitive Sciences, 13(11), 479-87.

Leary, M.R. (1990). Responses to social exclusion: social anxiety, jealousy, loneliness, depression, and low self-esteem. Journal of Social and Clinical Psychology, 9(2), 221-9.

Leary, M.R., Kowalski, R.M., Smith, L., Phillips, S. (2003). Teasing, rejection, and violence: case studies of the school shootings. Aggressive Behavior, 29(3), 202-14.

Leary, M.R., Twenge, J.M., Quinlivan, E. (2006). Interpersonal rejection as a determinant of anger and aggression. Personality and Social Psychology Review, 10(2), 111-32.

Levy, B.J., Wagner, A.D. (2011). Cognitive control and right ventrolateral prefrontal cortex: reflexive reorienting, motor inhibition, and action updating. Annals of the New York Academy of Sciences, 1224(1), 40-62.

Lopez, R.B., Hofmann, W., Wagner, D.D., Kelley, W.M., Heatherton, T.F. (2014). Neural predictors of giving in to temptation in daily life. Psychological Science, 25(7), 1337-44.

Lotze, M., Veit, R., Anders, S., Birbaumer, N. (2007). Evidence for a different role of the ventral and dorsal medial prefrontal cortex for social reactive aggression: an interactive fMRI study. NeuroImage, 34(1), 470-8.

MacDonald, G., Leary, M.R. (2005). Why does social exclusion hurt? The relationship between social and physical pain. Psychological Bulletin, 131(2), 202-23.

MacKinnon, D.P., Krull, J.L., Lockwood, C.M. (2000). Equivalence of the mediation, confounding and suppression effect. Prevention Science, 1(4), 173-81.

Maldjian, J.A., Laurienti, P.J., Kraft, R.A., Burdette, J.H. (2003). An automated method for neuroanatomic and cytoarchitectonic atlas-based interrogation of fMRI data sets. NeuroImage, 19(3), 1233-9.

McCullough, M.E., Kurzban, R., Tabak, B.A. (2013). Putting revenge and forgiveness in an evolutionary context. Behavioral and Brain Sciences, 36(1), 41-58.

Poldrack, R.A. (2006). Can cognitive processes be inferred from neuroimaging data?. Trends in Cognitive Sciences, 10(2), 59-63.

Poon, K.T., Teng, F. (2017). Feeling unrestricted by rules: ostracism promotes aggressive responses. Aggressive Behavior, 43(6), 558-67.

Price, D.D. (2000). Psychological and neural mechanisms of the affective dimension of pain. Science, 288(5472), 1769-72.

Rajchert, J., Winiewski, M. (2016). The behavioral approach and inhibition systems' role in shaping the displaced and direct aggressive reaction to ostracism and rejection. Personality and Individual Differences, 88(1), 272-9.

Ren, D., Wesselmann, E.D., Williams, K.D. (2018). Hurt people hurt people: ostracism and aggression. Current Opinion in Psychology, 19(1), 34-8.

Richeson, J.A., Baird, A.A., Gordon, H.L., et al. (2003). An fMRI investigation of the impact of interracial contact on executive function. Nature Neuroscience, 6(12), 1323-8.

Rotge, J.Y., Lemogne, C., Hinfray, S., et al. (2014). A meta-analysis of the anterior cingulate contribution to social pain. Social Cognitive and Affective Neuroscience, 10(1), 19-27.

Sabatinelli, D., Bradley, M.M., Lang, P.J., Costa, V.D., Versace, F. (2007). Pleasure rather than salience activates human nucleus accumbens and medial prefrontal cortex. Journal of Neurophysiology, 98(3), 1374-9.

Sandstrom, M.J., Cillessen, A.H., Eisenhower, A. (2003). Children's appraisal of peer rejection experiences: impact on social and emotional adjustment. Social Development, 12(4), 530-50.

Siemer, M., Mauss, I., Gross, J.J. (2007). Same situation-different emotions: how appraisals shape our emotions. Emotion, 7(3), 592.

Smith, S.M., Jenkinson, M., Woolrich, M.W., et al. (2004). Advances in functional and structural MR image analysis and implementation as FSL. NeuroImage, 23(1), S208-19.

Teneback, C.C., Nahas, Z., Speer, A.M., et al. (1999). Changes in prefrontal cortex and paralimbic activity in depression following two weeks of daily left prefrontal TMS. Journal of Neuropsychiatry and Clinical Neurosciences, 11(4), 426-35.

Twenge, J.M., Baumeister, R.F., Tice, D.M., Stucke, T.S. (2001). If you can't join them, beat them: effects of social exclusion on aggressive behavior. Journal of Personality and Social Psychology, 81(6), 1058-69.

Tzourio-Mazoyer, N., Landeau, B., Papathanassiou, D., et al. (2002). Automated anatomical labeling of activations in SPM using a macroscopic anatomical parcellation of the MNI MRI single-subject brain. NeuroImage, 15(1), 273-89.

Wager, T.D., Davidson, M.L., Hughes, B.L., Lindquist, M.A., Ochsner, K.N. (2008). Prefrontal-subcortical pathways mediating successful emotion regulation. Neuron, 59(6), 1037-50.

Wagner, D.D., Altman, M., Boswell, R.G., Kelley, W.M., Heatherton, T.F. (2013). Self-regulatory depletion enhances neural responses to rewards and impairs top-down control. Psychological Science, 24(11), 2262-71.

Warburton, W.A., Williams, K.D., Cairns, D.R. (2006). When ostracism leads to aggression: the moderating effects of control deprivation. Journal of Experimental Social Psychology, 42(2), 213-20.

Webster, G.D., DeWall, C.N., Pond, R.S., et al. (2014). The brief aggression questionnaire: psychometric and behavioral evidence for an efficient measure of trait aggression. Aggressive Behavior, 40(2), 120-39.

Wesselmann, E.D., Butler, F.A., Williams, K.D., Pickett, C.L. (2010). Adding injury to insult: unexpected rejection leads to more aggressive responses. Aggressive Behavior, 36(4), 232-7.

Williams, K.D. (2009). Ostracism: A temporal need-threat model. In: Zanna, M.P., editor. Advances in Experimental Social Psychology,, New York, NY: Academic Press.

Williams, K.D., Cheung, C.K., Choi, W. (2000). Cyberostracism: effects of being ignored over the Internet. Journal of Personality and Social Psychology, 79(5), 748-62.

Woolrich, M.W., Jbabdi, S., Patenaude, B., et al. (2009). Bayesian analysis of neuroimaging data in FSL. NeuroImage, 45(Suppl 1), S173-86.

Worsley, K.J. (2001). Statistical analysis of activation images. Functional MRI: An Introduction to Methods, 14(1), 251-70.

Yanagisawa, K., Masui, K., Onoda, K., et al. (2011). The effects of the behavioral inhibition and activation systems on social inclusion and exclusion. Journal of Experimental Social Psychology, 47(2), 502-5. 